\title{
Sign-reversing orbital polarization in the nematic phase of FeSe due to the $C_{2}$ symmetry-breaking in the self-energy
}

\author{
Seiichiro ONARI ${ }^{1,2}$, Youichi YAMAKaWA ${ }^{3}$, and Hiroshi KOnTANI ${ }^{3}$ \\ 1 Department of Physics, Okayama University, Okayama 700-8530, Japan \\ 2 Research Institute for Interdisciplinary Science, Okayama University \\ 3 Department of Physics, Nagoya University, Furo-cho, Nagoya 464-8602, Japan
}

(Dated: June 15, 2021)

\begin{abstract}
To understand the nematicity in Fe-based superconductors, nontrivial $\boldsymbol{k}$-dependence of the orbital polarization $\left(\Delta E_{x z}(\boldsymbol{k}), \Delta E_{y z}(\boldsymbol{k})\right)$ in the nematic phase, such as the sign reversal of the orbital splitting between $\Gamma$ - and $\mathrm{X}, \mathrm{Y}$-points in FeSe, provides significant information. To solve this problem, we study the spontaneous symmetry breaking with respect to the orbital polarization and spin susceptibility self-consistently. In FeSe, due to the sign-reversing orbital order, the hole- and electron-pockets are elongated along the $k_{y}$ - and $k_{x}$-axes respectively, consistently with experiments. In addition, an electron-pocket splits into two Dirac cone Fermi pockets with increasing the orbital polarization. The orbital-order in Fe-based superconductors originates from the strong positive feedback between the nematic orbital order and spin susceptibility.
\end{abstract}

PACS numbers: 74.70.Xa, 75.25.Dk, 74.20.Pq

The spontaneous symmetry breaking from $C_{4^{-}}$to $C_{2}{ }^{-}$ symmetry, so called the electronic nematic transition, is one of the fundamental unsolved electronic properties in Fe-based superconductors. To explain this nematicity, both the spin-nematic scenario [1-6] and the orbital order scenario [7-13] have been studied intensively. Above the structural transition temperatures $T_{\mathrm{str}}$, large enhancement of the electronic nematic susceptibility predicted by both scenarios $[1,10]$ is actually observed by the measurements of the softening of the shear modulus $C_{66}$ $[1,10,14,15]$, Raman spectroscopy, [16-18], and in-plane resistivity anisotropy $\Delta \rho[19]$.

To investigate the origin of the nematicity, FeSe $\left(T_{\mathrm{c}}=\right.$ $9 \mathrm{~K}$ ) is a favorable system since the electronic nematic state without magnetization is realized below $T_{\text {str }}=90$ $\mathrm{K}$ down to $0 \mathrm{~K}$. Above $T_{\text {str }}$, the antiferromagnetic fluctuations is weak and $T$-independent according to the NMR [20,21] and neutron scattering [22-24] studies, in contrast to the sizable spin fluctuations above $T_{\text {str }}$ in $\mathrm{LaFeAsO}[25]$ and $\mathrm{BaFe}_{2} \mathrm{As}_{2}$ [26]. This fact means that the magnetic instability is not a necessary condition for the electronic nematic state. In contrast to the smallness of the spin fluctuations, large nematic susceptibility is measured by $C_{66}$ and $\Delta \rho$ in FeSe. Based on the orbitalspin fluctuation theory, called the self-consistent vertexcorrection (SC-VC) theory, the development of the strong orbital fluctuations in FeSe are explained even when the spin-fluctuations are very small, consistently with experimental reports in FeSe [27]: The strong orbital fluctuations originate from the Aslamazov-Larkin vertex correction (AL-VC) that describes the orbital-spin modecoupling [10]. The nematic CDW in cuprate superconductor also originates from the AL-VC [28, 29].

The nontrivial electronic state below $T_{\text {str }}$ gives a crucial test for the theories proposed so far. In the orthorhombic phase with $(a-b) /(a+b) \sim 0.3 \%$, large orbital-splitting
$\left|E_{x z}-E_{y z}\right|$ of order $50 \mathrm{meV}$ is observed at X,Y-points by ARPES studies in $\mathrm{BaFe}_{2} \mathrm{As}_{2}$ [30], NaFeAs [31], and FeSe [32-40]. Especially, noticeable deformation of the Fermi surfaces (FSs) with $C_{2}$-symmetry is realized in FeSe, because of the smallness of the Fermi momenta. In FeSe, Ref. [38] reports that the orbital splitting $E_{x z}-E_{y z}$ is positive at $\Gamma$-point, whereas it is negative at $\mathrm{X}, \mathrm{Y}$-points. This sign-reversing orbital splitting is not realized in the non-magnetic orthorhombic phase in NaFeAs [31]. In addition, the e-FS1 at X-point is deformed to two Dirac cone Fermi pockets in thin-film FeSe [37, 40]. The aim of this study is to explain these nontrivial electronic states in the orbital-ordered states based on the realistic multiorbital Hubbard model.

Microscopically, the orbital order is expressed by the symmetry breaking in the self-energy. In the mean-field level approximations, however, the self-energy is constant in $\boldsymbol{k}$-space unless large inter-site Coulomb interactions are introduced [13]. For this reason, we have to study the non-local correlation effect beyond the mean-field theory, based on the realistic Hubbard model with onsite Coulomb interaction. We will show that the strong positive feedback between the nematic orbital order and $C_{2}$-symmetric spin susceptibility plays the essential role.

In this paper, we study the origin of the orbital order in Fe-based superconductors, by considering the cooperative symmetry breaking between the self-energy and spin susceptibility self-consistently. Experimentally observed strong $\boldsymbol{k}$-dependent orbital polarization $\left(\Delta E_{x z}(\boldsymbol{k}), \Delta E_{y z}(\boldsymbol{k})\right)$ is given by the non-local self-energy with $C_{2}$-symmetry. In the FeSe model, we obtain the sign-reversing orbital-splitting $E_{x z}-E_{y z}$ between $\Gamma$-point and X,Y-points reported in Refs. [38]. In addition, two Dirac-cone Fermi pockets emerge around X-point when the Coulomb interaction is larger [37, 40]. Thus, important key experimental electronic properties in the orbital- 
ordered phase are satisfactorily explained.

Hereafter, we denote the five $d$-orbital $d_{3 z^{2}-r^{2}}, d_{x z}$, $d_{y z}, d_{x y}, d_{x^{2}-y^{2}}$ as $l=1,2,3,4,5$. We study the realistic eight-orbital $d-p$ Hubbard models

$$
H_{\mathrm{M}}(r)=H_{\mathrm{M}}^{0}+r H_{\mathrm{M}}^{U} \quad(\mathrm{M}=\mathrm{LaFeAsO} \text { and FeSe }),
$$

where $H_{\mathrm{M}}^{U}$ is the first-principles screened Coulomb potential for $d$-orbitals in Ref. [41]: The averaged Coulomb interaction $\bar{U} \equiv \frac{1}{5} \sum_{l=1}^{5} U_{l}=7.21 \mathrm{eV}$ for FeSe is much larger than $\bar{U}=4.23 \mathrm{eV}$ for $\mathrm{LaFeAsO}$, since the number of the screening bands, by which $\bar{U}$ is reduced, is small in FeSe [41]. In contrast, the averaged Hund's coupling $\bar{J} \equiv \frac{1}{10} \sum_{l>m} J_{l, m}$ is similar in all compounds since the screening on the Hund's coupling is small. For this reason, the ratio $\bar{J} / \bar{U}=0.0945$ in FeSe is much smaller than the ratio $\bar{J} / \bar{U}=0.134$ in LaFeAsO. The factor $r(<1)$ is introduced to adjust the spin fluctuation strength.

$H_{\mathrm{M}}^{0}$ in Eq. (1) is the first-principle tight-binding model introduced in Ref. [27]. The band dispersion $E_{\boldsymbol{k}}^{\alpha}$ is the solution of $\operatorname{det}\left(\hat{z}^{-1} \epsilon+\mu-\hat{h}_{M}^{0}(\boldsymbol{k})\right)=0$, where $\hat{h}_{\mathrm{M}}^{0}(\boldsymbol{k})$ is the kinetic term and $\hat{z}^{-1}$ is the diagonal mass-enhancement factor: $\left(\hat{z}^{-1}\right)_{l, l^{\prime}} \equiv 1 / z_{l} \cdot \delta_{l, l^{\prime}}$. For LaFeAsO, we put $\hat{z}^{-1}=\hat{1}$. For FeSe, we put $1 / z_{4}=1.6$ and $1 / z_{l}=1$ $(l \neq 4)$ to represent the strong renormalization of the $d_{x y}$-orbital band [32]. Figures 1 show the bandstructures and the FSs in the FeSe and $\mathrm{LaFeAsO}$ models with $n_{\text {tot }}=2 \sum_{\alpha, \boldsymbol{k}} f\left(E_{\boldsymbol{k}}^{\alpha}\right)=12$. In FeSe, each Fermi pocket is very shallow [42]. The detail explanation for $H_{\mathrm{M}}^{0}$ is given in the Supplemental Material (SM) A [43].
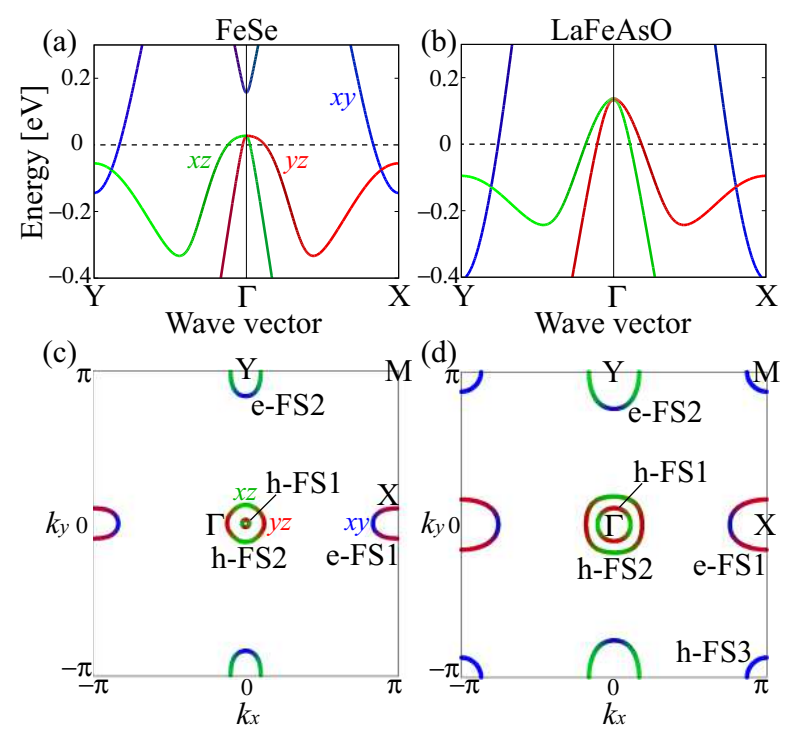

FIG. 1: (color online) Bandstructures of the eight-orbital models for (a) FeSe and (b) LaFeAsO in the unfolded Brillouin zone at $T=50 \mathrm{meV}$. FSs for the FeSe and LaFeAsO models are shown in (c) and (d), respectively. The colors correspond to 2 (green), 3 (red), and 4 (blue), respectively.

In this paper, we calculate the $\boldsymbol{k}$-dependence of the self-energy using the self-consistent one-loop approxima- tion, which have been applied to various single-orbital models [45] and multi-orbital models [12] in literature. The Green function in the orbital basis is

$$
\hat{G}(k)=\left(\hat{z}^{-1} i \epsilon_{n}+\mu-\hat{h}_{\mathrm{M}}^{0}(\boldsymbol{k})-\Delta \hat{\Sigma}(\boldsymbol{k})\right)^{-1},
$$

where $k=\left(\boldsymbol{k}, \epsilon_{n}=(2 n+1) \pi T\right)$ and $\Delta \hat{\Sigma}(\boldsymbol{k})$ is symmetry breaking self-energy. and $\hat{z}^{-1}$ is the diagonal massenhancement factor. The self-energy in the one-loop approximation $[12,45]$ is given as

$$
\Sigma_{l, l^{\prime}}(k)=\Sigma_{l, l^{\prime}}^{\mathrm{H}}+T \sum_{q, m, m^{\prime}} V_{l, m ; l^{\prime}, m^{\prime}}(q) G_{m, m^{\prime}}(k-q),
$$

where $\Sigma_{l, l^{\prime}}^{\mathrm{H}}=-\sum_{m, m^{\prime}} \Gamma_{l, l^{\prime} ; m^{\prime}, m}^{c} \Delta n_{m, m^{\prime}}$ is the Hartree term: $\Delta n_{m, m^{\prime}} \equiv\left\langle c_{i, m, \sigma}^{\dagger} c_{i, m^{\prime}, \sigma}\right\rangle-\left\langle c_{i, m, \sigma}^{\dagger} c_{i, m^{\prime}, \sigma}\right\rangle_{0}$. The non-local interaction $\hat{V}(q)$ in Eq. (3) is given as $\frac{3}{2} \hat{\Gamma}^{s} \hat{\chi}^{s}(q) \hat{\Gamma}^{s}+\frac{1}{2} \hat{\Gamma}^{c} \hat{\chi}^{c}(q) \hat{\Gamma}^{c}-\frac{1}{2}\left[\hat{\Gamma}^{c} \hat{\chi}^{0}(q) \hat{\Gamma}^{c}+\hat{\Gamma}^{s} \hat{\chi}^{0}(q) \hat{\Gamma}^{s}-\right.$ $\left.\frac{1}{4}\left(\hat{\Gamma}^{s}+\hat{\Gamma}^{c}\right) \hat{\chi}^{0}(q)\left(\hat{\Gamma}^{s}+\hat{\Gamma}^{c}\right)\right]$, where $\hat{\chi}^{s, c}(q)=\hat{\chi}^{0}(q)(1-$ $\left.\hat{\Gamma}^{s, c} \hat{\chi}^{0}(q)\right)^{-1}$ and $\chi_{l, l^{\prime}, m, m^{\prime}}^{0}(q)=-T \sum_{k} G_{l, m}(k+$ q) $G_{m^{\prime}, l^{\prime}}(k)$. Here, $\hat{\Gamma}^{c(s)}$ is the bare Coulomb interaction for the charge (spin) channel given in the SM A [43].

From Eq. (3), the $B_{1 g}$-type symmetry breaking selfenergy included in Eq. (2), which is orbital-diagonal, is derived as

$$
\Delta \Sigma_{l}(\boldsymbol{k})=\operatorname{Re}\left\{\Sigma_{l, l}\left(\boldsymbol{k}, \epsilon_{n}\right)-\Sigma_{l}^{\mathrm{A}_{1 \mathrm{~g}}}\left(\boldsymbol{k}, \epsilon_{n}\right)\right\}_{i \epsilon_{n} \rightarrow 0}
$$

for $l=2,3$, where $\Sigma_{l}^{\mathrm{A}_{1 \mathrm{~g}}}(k) \equiv\left(\Sigma_{l, l}(k)+\Sigma_{5-l, 5-l}\left(k^{\prime}\right)\right) / 2$ $\left(\boldsymbol{k}^{\prime}=\left(k_{y}, k_{x}\right)\right)$ is the $\mathrm{A}_{1 \mathrm{~g}}$-component of the self-energy. In the present study, we calculate Eqs. (3)-(4) selfconsistently [46]. We will show that $\Delta \Sigma_{l}$ emerges due to the strong positive feedback between the nematic orbital order and $C_{2}$-symmetric spin susceptibility: That is, near the nematic transition, infinitesimally small nematic orbital order enhances the spin susceptibility at $\boldsymbol{q}=(\pi, 0)$, and the enhanced spin-fluctuation-mediated interaction $\hat{V}(q)$ in turn enlarges the orbital order.

First, we study the FeSe model, using $64 \times 64 \boldsymbol{k}$ meshes and 512 Matsubara frequencies at $T=50 \mathrm{meV}$. In FeSe, the orbital order with $\Delta E_{x z}(\boldsymbol{k}) \equiv \Delta \Sigma_{2}(\boldsymbol{k})$ and $\Delta E_{y z}(\boldsymbol{k}) \equiv \Delta \Sigma_{3}(\boldsymbol{k})$ emerges when the spin Stoner factor $\alpha_{S}$, which is the maximum eigenvalue of $\hat{\Gamma}^{s} \hat{\chi}^{0}(\boldsymbol{q})$, is larger than $0.82(r>0.253)$. The magnetic order is realized when $\alpha_{S}=1$. In Figs. 2 (a) and (b), we show respectively the obtained orbital polarization $\left(\Delta E_{x z}(\boldsymbol{k}), \Delta E_{y z}(\boldsymbol{k})\right)$ and the spin susceptibility $\chi^{s}(\boldsymbol{q}) \equiv \sum_{l, m} \chi_{l, l ; m, m}^{s}(\boldsymbol{q})$ for $\alpha_{S}=0.868$. Due to the positive $\Delta E_{y z}(\mathrm{X})$, the e-FS1 around X-point is modified to the pair of the Dirac-cone Fermi pockets. Another electron-pocket around Y-point, e-FS2, is enlarged by the negative $\Delta E_{x z}(\mathrm{Y})$. We stress that $\Delta E_{x z(y z)}(\boldsymbol{k})$ changes its sign along the $k_{y(x)}$-axis shown by the broken line in Fig. 2 (a). Due to this sign reversal, the outer 

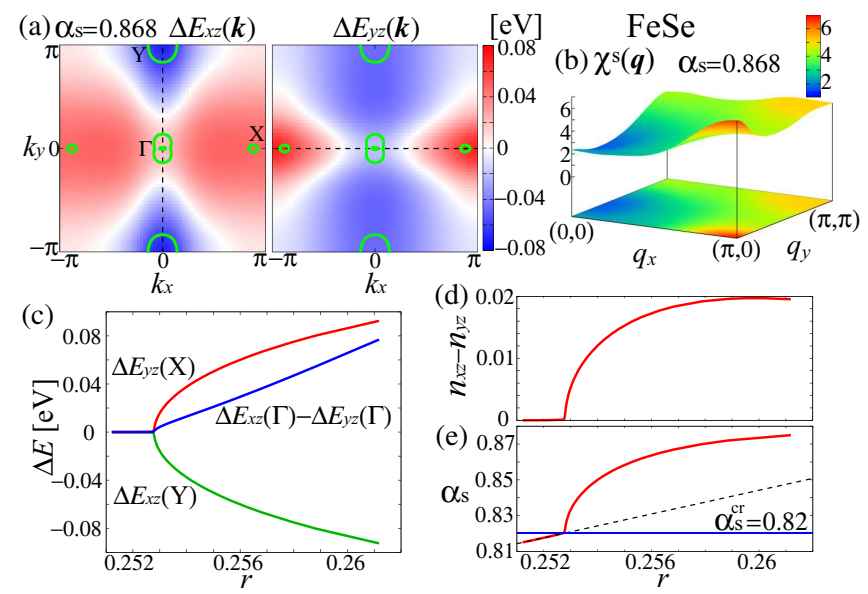

FIG. 2: (color online) (a) Orbital sign-reversing polarization $\left(\Delta E_{x z}(\boldsymbol{k}), \Delta E_{y z}(\boldsymbol{k})\right)$ and $(\mathrm{b}) \chi^{s}(\boldsymbol{q})$ in the FeSe model for $\alpha_{S}=0.868(r=0.257)$. The realized FSs are shown by the green lines. The splitting $E_{x z}-E_{y z}$ at $\Gamma$-point is positive, as observed in Ref. [38]. (c) The polarization at $\Gamma-, \mathrm{X}$ - and Ypoints. (d) $\Delta n \equiv n_{x z}-n_{y z}$ and (e) $\alpha_{S}$ for $0.261>r>0.251$.

hole-pocket (h-FS2) is elongated along the $k_{y}$-axis, as observed experimentally [38]. Due to the orbital polarization, $\alpha_{S}$ increases and $\chi^{s}(\boldsymbol{q})$ shows the $C_{2}$ anisotropy $\chi^{s}(\pi, 0)>\chi^{s}(0, \pi)$ shown in Fig. 2 (b) $[6,47]$.

In Fig. 2 (c), we show the $r$-dependences of the orbital polarization at $\Gamma$-, $\mathrm{X}$ - and $\mathrm{Y}$-points. With increasing $r$, the spin-fluctuation-driven orbital order appears as a second-order transition at $\alpha_{S}^{\mathrm{cr}}=0.82$. The relations $\Delta E_{x z}(\mathrm{Y})<0$ and $\Delta E_{y z}(\mathrm{X})>0$ hold in the ordered state. In FeSe, the orbital splitting $E_{x z}-E_{y z}$ at $\Gamma$-point is positive, so h-FS2 is elongated along the $k_{y}$-axis. Such sign-reversing orbital order does not occur in the LaFeAsO model (see Fig. 5). Since $d_{x z^{-}}$and $d_{y z}$-orbitals are exchanged by $\pi / 2$-rotation, the obtained order $\left(\Delta E_{x z}(\boldsymbol{k}), \Delta E_{y z}(\boldsymbol{k})\right)$ belongs to the $B_{1 g}$ representation (=d-wave), in spite of $\Delta E_{x z}(\boldsymbol{k}) \neq \Delta E_{y z}(\boldsymbol{k})[13,36]$.

Figure 2 (d) shows the difference $\Delta n \equiv n_{x z}-n_{y z}$ : $\Delta n \sim+10^{-2}$ will induce the small lattice deformation $(a-b) /(a+b) \approx 0.2 \%$ in FeSe due to small $e$-ph interaction. When $\Delta n \neq 0$, the spin Stoner factor $\alpha_{S}$ is strongly enlarged as shown in Fig. 2 (e), consistently with the enhancement of spin fluctuations observed by NMR [20, 21] and neutron [22, 23] measurements. For a fixed $r, \Delta n$ shows a mean-field-type second-order $T$ dependence below $T_{\text {str }}[27]$.

In Fig. 3, we display the $C_{2}$ bandstructures obtained in the FeSe model for (a) $\alpha_{S}=0.854$ and (b) $\alpha_{S}=0.868$. In both cases, $\Delta E_{y z}\left(k_{x}, 0\right)$ and $\Delta E_{x z}\left(0, k_{y}\right)$ show sign reversal, and $E_{x z}-E_{y z}>0$ at $\Gamma$-point. The shape of the FSs in Fig. 3 (c) for $\alpha_{S}=0.854$ (Fig. 3 (d) for $\alpha_{S}=0.868$ ) is consistent with the FSs observed in bulk FeSe (thin-film $\mathrm{FeSe}$ ). This result indicates that the electron correlation in thin-film FeSe is slightly stronger, consistently with
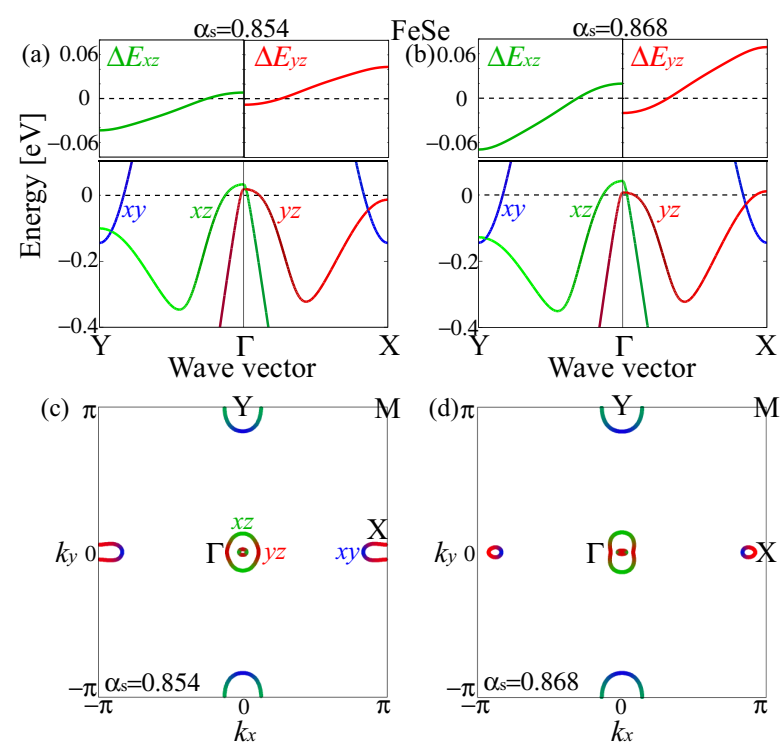

FIG. 3: (color online) Orbital polarizations and bandstructures in the FeSe model along $\mathrm{Y} \rightarrow \Gamma \rightarrow \mathrm{X}$ for (a) $\alpha_{S}=0.854$ and (b) $\alpha_{S}=0.868$. The corresponding FSs at $T=50 \mathrm{meV}$ are shown in (c) and (d), respectively.

the higher $T_{\text {str }} \approx 120 \mathrm{~K}$ in thin-film FeSe.

In real FeSe, the inner pocket sinks under the Fermi level due to the spin-orbit interaction. Similar single hole-FS model is introduced in the SM B [43] by shifting the $d_{x y}$-orbital level at $\Gamma$-point. It is verified that the sign-reversing orbital polarization emerges in the FeSe model with single hole-FS in the SM B [43].

Here, we explain that the origin of the orbital order is the positive feedback between the orbital polarization $\left(\Delta E_{x z}(\boldsymbol{k}), \Delta E_{y z}(\boldsymbol{k})\right)$ and the spin susceptibility. For this purpose, we introduce the following two simplified selfenergies and perform the self-consistent calculations:

$$
\begin{aligned}
& \Sigma_{l, l^{\prime}}^{\mathrm{AL}}(k)=\Sigma_{l, l^{\prime}}^{\mathrm{H}}+T \sum_{q, m, m^{\prime}} V_{l, m ; l^{\prime}, m^{\prime}}(q) G_{m, m^{\prime}}^{\Delta E=0}(k-q),(5) \\
& \Sigma_{l, l^{\prime}}^{\mathrm{MT}}(k)=\Sigma_{l, l^{\prime}}^{\mathrm{H}}+T \sum_{q, m, m^{\prime}} V_{l, m ; l^{\prime}, m^{\prime}}^{\Delta E=0}(q) G_{m, m^{\prime}}(k-q),(6)
\end{aligned}
$$

where the superscript " $\Delta E=0$ " means the absence of the $C_{2}$ orbital polarization. In Eq. (5) (Eq. (6)), only the feedback effect from the symmetry-breaking spin susceptibility (Green function) is included. $\hat{\Sigma}^{\mathrm{AL}(\mathrm{MT})}$ contains the Aslamazov-Larkin term (Maki-Thompson term) that is the second-order (first-order) term with respect to $\chi^{s}$ in Fig. 4 (a), which shows the expansion of the self-energy in Eq. (3) by using the relation $\hat{G}=\hat{G}^{\Delta \mathrm{E}=0}+\hat{G}^{\Delta \mathrm{E}=0} \cdot \Delta \hat{E} \cdot \hat{G}^{\Delta \mathrm{E}=0}+O\left(\Delta E^{2}\right)$; see the SM C [43] for detailed explanation. Figure 4 (b) shows the orbital polarizations $\Delta E_{y z}^{\mathrm{AL}}$ and $\Delta E_{y z}^{\mathrm{MT}}$ at $\mathrm{X}$-point derived from Eqs. (5) and (6), respectively, as functions of $r$ at $T=50 \mathrm{meV}$. Here, $\Delta E_{y z}^{\text {all }}$ is the orbital polarization derived from Eq. (3), shown in Fig. 2 (c). The 
closeness of $\Delta E_{y z}^{\mathrm{AL}}$ and $\Delta E_{y z}^{\mathrm{all}}$ means that the orbital order is mainly driven by the Aslamazov-Larkin term $\hat{\Sigma}^{\mathrm{AL}}$. Figure 4 (c) shows that $\alpha_{S}$ is strongly enlarged by the orbital order due to $\hat{\Sigma}^{\mathrm{AL}}$, similarly to $\alpha_{S}$ in Fig. 2 (e). In contrast, $\alpha_{S}$ in Fig. 4 (d) is suppressed because of the wrong $\boldsymbol{q}=(\pi, 0)$ nesting on the $d_{y z}$-orbital due to $\hat{\Sigma}^{\mathrm{MT}}$. Thus, the origin of the orbital order is the strong positive feedback between the orbital polarization and $C_{2}$ spin susceptibility described by the Aslamazov-Larkin term in Eq. (5).

Although $\hat{\Sigma}^{\mathrm{AL}}$ is the main driving force of the orbital order, the hole-pocket is elongated along the $k_{x}$-axis in the ordered state driven by $\hat{\Sigma}^{\mathrm{AL}}$ as shown in the left inset of Fig. 4 (b). Therefore, the Maki-Thompson term is indispensable to reproduce the sign-reversing orbital polarization; see the right inset of Fig. 4 (b). In SM C [43], we explain analytically why the Maki-Thompson term gives the sign reversal. Thus, although $\Delta E_{y z}^{\mathrm{AL}}\left(k_{x}, 0\right)$ is always positive as shown in the left inset of Fig. 4 (b), $\Delta E_{y z}^{\text {all }}\left(k_{x}, 0\right)$ in Fig. 2 (a) given by Eq. (3) changes to negative at $k_{x} \sim 0$, due to the sign-reversing MakiThompson term that is small in magnitude at $\alpha_{S} \gtrsim \alpha_{S}^{\mathrm{cr}}$.
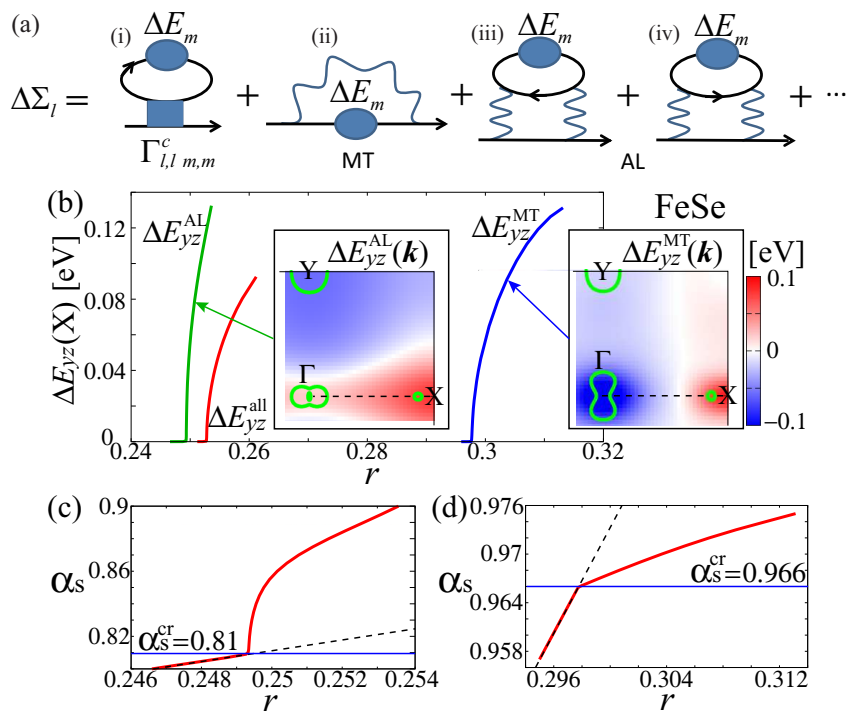

FIG. 4: (color online) (a) Expansion of the self-energy with respect to $\Delta E$. The MT term (AL term) is included in $\hat{\Sigma}^{\mathrm{MT}(\mathrm{AL})}$. (b) $r$-dependences of $\Delta E_{y z}^{\mathrm{AL}}$ and $\Delta E_{y z}^{\mathrm{MT}}$ at X-point. $\Delta E_{y z}^{\text {all }}$ is the orbital polarization derived from Eq. (3) shown in Fig. 2 (c). (c) $\alpha_{S}$ due to $\hat{\Sigma}^{\mathrm{AL}}$, and (d) $\alpha_{S}$ due to $\hat{\Sigma}^{\mathrm{MT}}$.

Next, we show the numerical results for the $\mathrm{LaFeAsO}$ model with $\hat{z}^{-1}=\hat{1}$. Then, the orbital order is realized for $\alpha_{S}>0.91$. In Fig. 5 (a), we show the obtained orbital polarization at $\alpha_{S}=0.98$ and $T=47 \mathrm{meV}$. The e-FS1 around X-point is smaller than e-FS2 around Ypoint due to the orbital polarization $\Delta E_{x z}(\mathrm{Y})<0$ and $\Delta E_{y z}(\mathrm{X})>0$. In addition, $\Delta E_{y z}\left(k_{x}, 0\right)\left(\Delta E_{x z}\left(0, k_{y}\right)\right)$ is always positive (negative), and therefore the outer hole-pocket is elongated along the $k_{x}$-axis. This result is consistent with the Fermi surface deformation in the non-magnetic orthorhombic phase in NaFeAs [31]. In the ordered state, strong in-plane anisotropy of the spin susceptibility $\chi^{s}(\pi, 0) \gg \chi^{s}(0, \pi)$ is realized as shown in Fig. 5 (b) [47], consistently with the neutron scattering experiments. The bandstructure is shown in Fig. 5 (c).

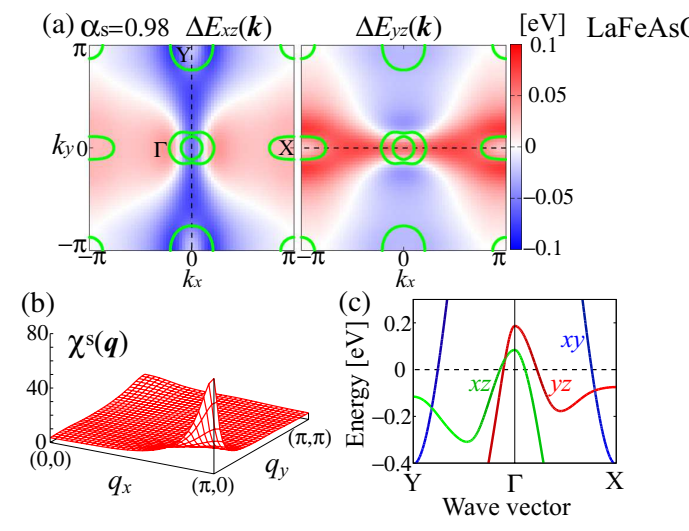

FIG. 5: (color online) (a) Obtained sign-preserving orbital polarization $\left(\Delta E_{x z}(\boldsymbol{k}), \Delta E_{y z}(\boldsymbol{k})\right)$ in the LaFeAsO model for $\alpha_{S}=0.98(r=0.376)$. The FSs are shown by the green lines. The corresponding spin susceptibility and the bandstructure are shown in (b) and (c), respectively.

In this study, we succeeded in explaining (i) the small critical Stoner factor $\alpha_{S}^{\mathrm{cr}}$ and (ii) the relation $E_{x z}>E_{y z}$ near $\Gamma$-point in FeSe. An origin of (i) is the smallness of the ratio $\bar{J} / \bar{U}$, as verified by the SC-VC theory [27] and the renormalization group (RG) theory $[48,49]$. Another origin of (i) is the smallness of the FSs in FeSe, since the three-point vertex $\hat{\Lambda}_{3} \equiv \delta \hat{\chi}^{0}(\boldsymbol{q}) / \delta \Delta \hat{E}$ in the Aslamazov-Larkin term increases when the particle-hole asymmetry is large [27]. The relation (ii) can be realized by the sign-reversing Maki-Thomson term. Although the Maki-Thompson term is usually small, (ii) is actually realized when the FSs are smaller since the minimum of the Aslamazov-Larkin term $\Delta E_{y z}^{\mathrm{AL}}(\boldsymbol{k})$ shifts to $\boldsymbol{k}=\mathbf{0}$. Recently, the advantage of the small FSs for the nematicity had been stressed by the RG study in Ref. [50].

In summary, we investigated the $C_{2}$ symmetry breaking in the self-energy and susceptibility self-consistently, and explained the experimental $C_{2}$-symmetric FSs and $\chi^{s}(\boldsymbol{q})$. In the FeSe model, experimental deformation of the FSs due to the sign-reversing orbital polarization is satisfactorily reproduced. In the LaFeAsO model, in contrast, spin fluctuations are strongly enlarged by the sign-preserving orbital polarization. Thus, the key experiments below $T_{\text {str }}$ are satisfactorily explained. The orbital order originates from the positive feedback between the nematic orbital order and spin susceptibility due to the Aslamazov-Larkin term, and the sign reversal of $\Delta E_{y z}\left(k_{x}, 0\right)$ and $\Delta E_{x z}\left(0, k_{y}\right)$ in FeSe is caused by the Maki-Thompson term.

We are grateful to Y. Matsuda, T. Shibauchi, T. Shi- 
mojima, A. Chubukov, J. Schmalian and R. Fernandes for useful discussions. This study has been supported by Grants-in-Aid for Scientific Research from MEXT of Japan.

[1] R. M. Fernandes, L. H. VanBebber, S. Bhattacharya, P. Chandra, V. Keppens, D. Mandrus, M. A. McGuire, B. C. Sales, A. S. Sefat, and J. Schmalian, Phys. Rev. Lett. 105, 157003 (2010).

[2] F. Wang, S. A. Kivelson, and D.-H. Lee, Nat. Phys. 11, 959 (2015).

[3] A. V. Chubukov, R. M. Fernandes, and J. Schmalian, Phys. Rev. B 91, 201105 (2015).

[4] R. Yu, and Q. Si, Phys. Rev. Lett. 115, 116401 (2015).

[5] J. K. Glasbrenner, I. I. Mazin, H. O. Jeschke, P. J. Hirschfeld, and R. Valenti, Nat. Phys. 11, 953 (2015).

[6] L. Fanfarillo, A. Cortijo, and B. Valenzuela, Phys. Rev B. 91, 214515 (2015).

[7] F. Krüger, S. Kumar, J. Zaanen, J. van den Brink, Phys. Rev. B 79, 054504 (2009).

[8] W. Lv, J. Wu, and P. Phillips, Phys. Rev. B 80, 224506 (2009).

[9] C.-C. Lee, W.-G. Yin, and W. Ku, Phys. Rev. Lett. 103, 267001 (2009).

[10] S. Onari and H. Kontani, Phys. Rev. Lett. 109, 137001 (2012).

[11] S. Onari, Y. Yamakawa, and H. Kontani, Phys. Rev. Lett. 112, 187001 (2014).

[12] S. Onari and H. Kontani, Iron-Based Superconductivity, (ed. P.D. Johnson, G. Xu, and W.-G. Yin, SpringerVerlag Berlin and Heidelberg GmbH \& Co. K (2015)).

[13] K. Jiang, J. P. Hu, H. Ding, and Z. Wang, Phys. Rev. B 93, 115138 (2016).

[14] M. Yoshizawa, D. Kimura, T. Chiba, S. Simayi, Y. Nakanishi, K. Kihou, C.-H. Lee, A. Iyo, H. Eisaki, M. Nakajima, and S. Uchida, J. Phys. Soc. Jpn. 81, 024604 (2012).

[15] A. E. Böhmer, P. Burger, F. Hardy, T. Wolf, P. Schweiss, R. Fromknecht, M. Reinecker, W. Schranz, and C. Meingast, Phys. Rev. Lett. 112, 047001 (2014).

[16] Y. Gallais, R. M. Fernandes, I. Paul, L. Chauviere, Y.-X. Yang, M.-A. Measson, M. Cazayous, A. Sacuto, D. Colson, and A. Forget, Phys. Rev. Lett. 111, 267001 (2013).

[17] H. Kontani and Y. Yamakawa, Phys. Rev. Lett. 113, 047001 (2014).

[18] M. Khodas, and A. Levchenko, Phys. Rev. B 91, 235119 (2015).

[19] J.-H. Chu, H.-H. Kuo, J. G. Analytis, and I. R. Fisher, Science 337, 710 (2012).

[20] A. E. Böhmer, T. Arai, F. Hardy, T. Hattori, T. Iye, T. Wolf, H. v. Löhneysen, K. Ishida, and C. Meingast, Phys. Rev. Lett. 114, 027001 (2015).

[21] S.-H. Baek, D. V. Efremov, J. M. Ok, J. S. Kim, Jeroen van den Brink, and B. Büchner, Nat. Mater. 14, 210 (2015).

[22] M. C. Rahn, R. A. Ewings, S. J. Sedlmaier, S. J. Clarke, and A. T. Boothroyd, Phys. Rev. B 91, 180501(R) (2015).

[23] Q. Wang, Y. Shen, B. Pan, Y. Hao, M. Ma, F. Zhou, P. Steffens, K. Schmalzl, T. R. Forrest, M. Abdel-Hafiez, D.
A. Chareev, A. N. Vasiliev, P. Bourges, Y. Sidis, H. Cao, and J. Zhao, Nat. Mater. 15, 159 (2016).

[24] S. Shamoto, K. Matsuoka, R. Kajimoto, M. Ishikado, Y. Yamakawa, T. Watashige, S. Kasahara, M. Nakamura, H. Kontani, T. Shibauchi, and Y. Matsuda, arXiv:1511.04267.

[25] Y. Nakai, S. Kitagawa, T. Iye, K. Ishida, Y. Kamihara, M. Hirano, and H. Hosono, Phys. Rev. B 85, 134408 (2012).

[26] F. L. Ning, K. Ahilan, T. Imai, A. S. Sefat, M. A. McGuire, B. C. Sales, D. Mandrus, P. Cheng, B. Shen, and H.-H Wen, Phys. Rev. Lett. 104, 037001 (2010).

[27] Y. Yamakawa, S. Onari and H. Kontani, arXiv:1509.01161.

[28] Y. Yamakawa and H. Kontani, Phys. Rev. Lett. 114, 257001 (2015).

[29] M. Tsuchiizu, Y. Yamakawa and H. Kontani, arXiv:1508.07218.

[30] M. Yi, D. Lu, J.-H. Chu, J. G. Analytis, A. P. Sorini, A. F. Kemper, B. Moritz, S.-K. Mo, R. G. Moore, M. Hashimoto, W.-S. Lee, Z. Hussain, T. P. Devereaux, I. R. Fisher, and Z.-X. Shen, Proc. Natl. Acad. Sci. USA 108, 6878 (2011).

[31] Y. Zhang, C. He, Z. R. Ye, J. Jiang, F. Chen, M. Xu, Q. Q. Ge, B. P. Xie,, J. Wei, M. Aeschlimann, X. Y. Cui, M. Shi, J. P. Hu, and D. L. Feng, Phys. Rev. B 85, 085121 (2012).

[32] J. Maletz, V. B. Zabolotnyy, D. V. Evtushinsky, S. Thirupathaiah, A. U. B. Wolter, L. Harnagea, A. N. Yaresko, A. N. Vasiliev, D. A. Chareev, A. E. Böhmer, F. Hardy, T. Wolf, C. Meingast, E. D. L. Rienks, B. Büchner, and S. V. Borisenko, Phys. Rev. B 89, 220506(R) (2014).

[33] K. Nakayama, Y. Miyata, G. N. Phan, T. Sato, Y. Tanabe, T. Urata, K. Tanigaki, and T. Takahashi, Phys. Rev. Lett. 113, 237001 (2014).

[34] M. D. Watson, T. K. Kim, A. A. Haghighirad, N. R. Davies, A. McCollam, A. Narayanan, S. F. Blake, Y. L. Chen, S. Ghannadzadeh, A. J. Schofield, M. Hoesch, C. Meingast, T. Wolf, and A. I. Coldea, Phys. Rev. B 91, 155106 (2015).

[35] T. Shimojima, Y. Suzuki, T. Sonobe, A. Nakamura, M. Sakano, J. Omachi, K. Yoshioka, M. Kuwata-Gonokami, K. Ono, H. Kumigashira, A. E. Böhmer, F. Hardy, T. Wolf, C. Meingast, H. v. Löhneysen, H. Ikeda, and K. Ishizaka, Phys. Rev. B 90, 121111(R) (2014).

[36] P. Zhang, T. Qian, P. Richard, X. P. Wang, H. Miao, B. Q. Lv, B. B. Fu, T. Wolf, C. Meingast, X. X. Wu, Z. Q. Wang, J. P. Hu, and H. Ding, Phys. Rev. B 91, 214503 (2015).

[37] Y. Zhang, M. Yi, Z.-K. Liu, W. Li, J. J. Lee, R. G. Moore, M. Hashimoto, N. Masamichi, H. Eisaki, S. -K. Mo, Z. Hussain, T. P. Devereaux, Z.-X. Shen, and D. H. Lu, arXiv:1503.01556.

[38] Y. Suzuki, T. Shimojima, T. Sonobe, A. Nakamura, M. Sakano, H. Tsuji, J. Omachi, K. Yoshioka, M. KuwataGonokami, T. Watashige, R. Kobayashi, S. Kasahara, T. Shibauchi, Y. Matsuda, Y. Yamakawa, H. Kontani, and K. Ishizaka, Phys. Rev. B 92, 205117 (2015).

[39] M. D. Watson, T. K. Kim, A. A. Haghighirad, S. F. Blake, N. R. Davies, M. Hoesch, T. Wolf, and A. I. Coldea, Phys. Rev. B 92, 121108 (2015).

[40] S. Y. Tan, Y. Fang, D. H. Xie, W. Feng, C. H. P. Wen, Q. Song, Q. Y. Chen, W. Zhang, Y. Zhang, L. Z. Luo, B. P. Xie, X. C. Lai, and D. L. Feng, Phys. Rev. B 93, 
104513 (2016).

[41] T. Miyake, K. Nakamura, R. Arita, and M. Imada, J. Phys. Soc. Jpn. 79, 044705 (2010).

[42] S. Kasahara, T. Watashige, T. Hanaguri, Y. Kohsaka, T. Yamashita, Y. Shimoyama, Y. Mizukami, R. Endo, H. Ikeda, K. Aoyama, T. Terashima, S. Uji, T. Wolf, H. v. Löhneysen, T. Shibauchi, Y. Matsuda, Proc. Natl. Acad. Sci. USA 46, 16309 (2014).

[43] Supplemental Material, which includes Ref. [44].

[44] T. Saito, Y. Yamakawa, S. Onari, and H. Kontani, Phys. Rev. B 92, 134522 (2015).

[45] N. E. Bickers and S. R. White, Phys. Rev. B 43, 8044 (1991).

[46] We verified that the ciritical value of $r$ for the orbital order is slightly enlarged if Eq. (4) is replaced with $\Delta \Sigma_{l}\left(\boldsymbol{k}, \epsilon_{n}\right)=\left\{\Sigma_{l, l}\left(k, \epsilon_{n}\right)-\Sigma_{l}^{\mathrm{A}_{1 \mathrm{~g}}}\left(\boldsymbol{k}, \epsilon_{n}\right)\right\}$.

[47] H. Kontani, T. Saito, and S. Onari, Phys. Rev. B 84, 024528 (2011).

[48] M. Tsuchiizu, Y. Ohno, S. Onari, and H. Kontani, Phys. Rev. Lett. 111, 057003 (2013).

[49] M. Tsuchiizu, Y. Yamakawa, S. Onari, Y. Ohno, and H. Kontani, Phys. Rev. B 91, 155103 (2015).

[50] A.V. Chubukov, M. Khodas, and R. M. Fernandes, arXiv:1602.05503. 


\title{
[Supplementary Material] \\ Sign-reversing orbital polarization in the nematic phase of FeSe due to the symmetry-breaking in self-energy
}

\author{
Seiichiro Onari ${ }^{1,2}$, Youichi Yamakawa ${ }^{3}$, and Hiroshi Kontani ${ }^{3}$ \\ 1 Department of Physics, Okayama University, Okayama 700-8530, Japan \\ 2 Research Institute for Interdisciplinary Science, Okayama University \\ 3 Department of Physics, Nagoya University, Nagoya 464-8602, Japan
}

\section{A: Eight-orbital models for FeSe and LaFeAsO, effect of the spin-orbit interaction}

We introduce the eight-orbital $d-p$ models for FeSe and LaFeAsO analyzed in the main text. We first derived the first principles tight-binding models using the WIEN2k and WANNIER90 codes. Next, in order to obtain the experimentally observed Fermi surfaces (FSs), we introduce the $\boldsymbol{k}$-dependent shifts for orbital $l, \delta E_{l}(\boldsymbol{k})$, by introducing the intra-orbital hopping parameters, as we explain in Ref. [27]. We shift the $d_{x y}$-orbital band $\left[d_{x z / y z^{-o r b i t a l}}\right.$ band $]$ at $(\Gamma, \mathrm{M}, \mathrm{X})$ points by $(0,-0.25,+0.24)[(-0.24$, $0,+0.12)]$ for FeSe, and $(0,0,+0.05)[(-0.05,0,+0.05)]$ for $\mathrm{LaFeAsO}$. The unit is $\mathrm{eV}$.

Next, we discuss the bandstructure in the presence of the spin-orbit interaction (SOI), which is expressed as $\lambda \sum_{i} \boldsymbol{l}_{i} \cdot \boldsymbol{\sigma}_{i}$. The matrix elements of $\boldsymbol{l}_{i}$ are given in Ref. [44]. To study the effect of the SOI, we have to use the ten-orbital model since the "unfolding" is prohibited by the SOI. In addition, the $\mathrm{SU}(2)$ symmetry for conduction electron spins is violated by the SOI. For these reason, in the presence of the SOI, the numerical study of the self-energy calculation becomes very difficult.

Figures S1 (a) and (b) show the bandstructures and hole-FSs for $\lambda=0$ and $\lambda=50 \mathrm{meV}$, respectively, in the FeSe model in the absence of orbital polarization. The main effect of the SOI on the hole-FSs is that the inner pocket shrinks or sinks below the Fermi level. In the presence of the orbital polarization $E_{x z}-E_{y z}=40 \mathrm{meV}$ at $\Gamma$-point, the bandstructures and hole-FSs are modified to Figs. S1 (c) for $\lambda=0$ and (d) for $\lambda=50 \mathrm{meV}$. In both cases, the outer hole-FS is mainly composed of the $d_{x z^{-}}$ orbital, which is consistent with the experimental report [38], when we set the radius of the hole-FS just slightly smaller than the present model $(\mu=+10 \mathrm{meV})$. The hollows of the hole-FS near the $k_{x}$-axis become smaller in the presence of the SOI.

Finally, the bare Coulomb interaction for the spin
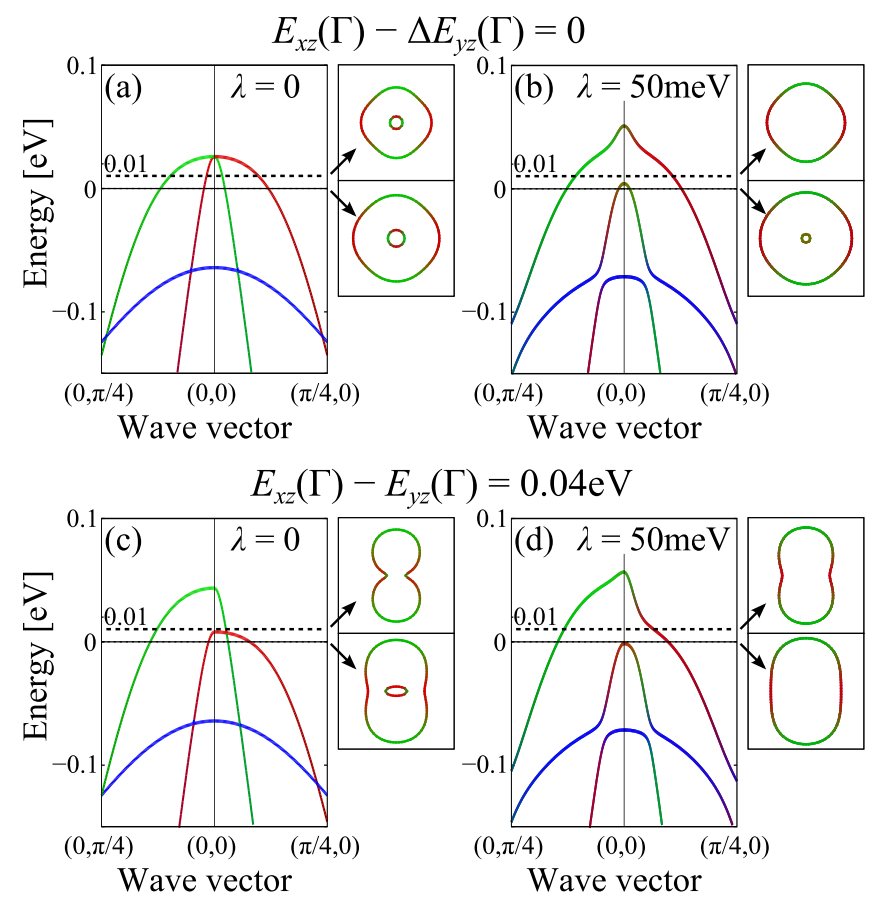

FIG. S1: (color online) Bandstructures and hole-FSs in the FeSe model. (a) $\lambda=0$ (without SOI) and (b) $\lambda=50 \mathrm{meV}$ (with SOI) for $E_{x z}-E_{y z}=0$ at $\Gamma$-point. (c) $\lambda=0$ (without SOI) and (d) $\lambda=50 \mathrm{meV}$ (with SOI) for $E_{x z}-E_{y z}=40$ meV at $\Gamma$-point.

channel in the main text is

$$
\left(\Gamma^{\mathrm{s}}\right)_{l_{1} l_{2}, l_{3} l_{4}}= \begin{cases}U_{l_{1}, l_{1}}, & l_{1}=l_{2}=l_{3}=l_{4} \\ U_{l_{1}, l_{2}}^{\prime}, & l_{1}=l_{3} \neq l_{2}=l_{4} \\ J_{l_{1}, l_{3}}, & l_{1}=l_{2} \neq l_{3}=l_{4} \\ J_{l_{1}, l_{2}}, & l_{1}=l_{4} \neq l_{2}=l_{3} \\ 0, & \text { otherwise. }\end{cases}
$$

Also, the bare Coulomb interaction for the charge channel 
is

$$
\left(\hat{\Gamma}^{\mathrm{c}}\right)_{l_{1} l_{2}, l_{3} l_{4}}= \begin{cases}-U_{l_{1}, l_{1}}, & l_{1}=l_{2}=l_{3}=l_{4} \\ U_{l_{1}, l_{2}}^{\prime}-2 J_{1_{1}, l_{2}}, & l_{1}=l_{3} \neq l_{2}=l_{4} \\ -2 U_{l_{1}, l_{3}}^{\prime}+J_{l_{1}, l_{3}}, & l_{1}=l_{2} \neq l_{3}=l_{4} \\ -J_{1_{1}, l_{2}}, & l_{1}=l_{4} \neq l_{2}=l_{3} \\ 0 . & \text { otherwise. }\end{cases}
$$

Here, $U_{l, l}, U_{l, l^{\prime}}^{\prime}$ and $J_{l, l^{\prime}}$ are the first principles Coulomb interaction terms given in Ref. [41].

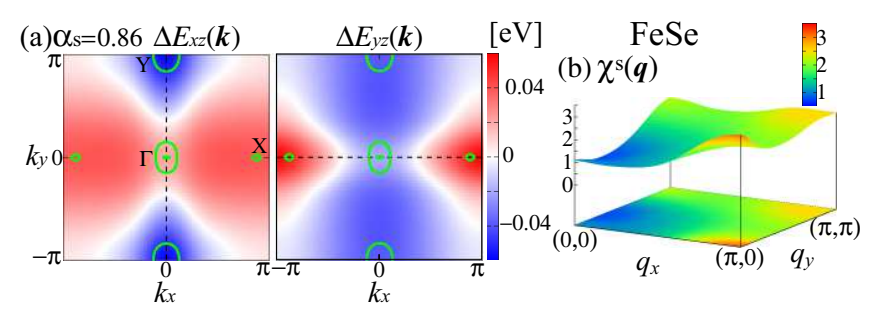

FIG. S2: (color online) (a) $\left(\Delta E_{x z}(\boldsymbol{k}), \Delta E_{y z}(\boldsymbol{k})\right)$ and (b) $\chi^{s}(\boldsymbol{q})$ in the FeSe model for $\alpha_{S}=0.860(r=0.506)$ at $T=25 \mathrm{meV}$ in the case of $z=1 / 2$. The obtained $C_{2}$-deformation of the FSs and that of $\chi^{s}(\boldsymbol{q})$ are very similar to Figs. 2 (a) and (b) in the main text.

In the main text, we put the renormalization factors as $\left(z_{1}, z_{2}, z_{3}, z_{4}, z_{5}\right)=(1,1,1,1 / 1.6,1)$. In the case of $\left(z_{1}, z_{2}, z_{3}, z_{4}, z_{5}\right)=z(1,1,1,1 / 1.6,1)$, we proved that in Ref. [27] that essentially same numerical results are obtained by replacing $r$ and $T$ with $r / z$ and $T z$, respectively. To verify this scaling relation, we perform the numerical study for $z=1 / 2$ : Figure $\mathrm{S} 2$ shows the obtained (a) $\left(\Delta E_{x z}(\boldsymbol{k}), \Delta E_{y z}(\boldsymbol{k})\right)$ and (b) $\chi^{s}(\boldsymbol{q})$ in the FeSe model for $z=1 / 2, r=0.506$ and $T=25 \mathrm{meV}$ $\left(\alpha_{S}=0.860\right)$. These results are very similar to those for $z=1, r=0.257$ and $T=50 \mathrm{meV}\left(\alpha_{S}=0.868\right)$ shown in Fig. 2 in the main text. Thus, the nematic orbital order is obtained by the present theory even if the band renormalization effect is taken into account.

In the present study, we neglected the damping of the quasiparticle given by the imaginary part of the selfenergy. We expect that this effect becomes small at sufficiently low temperatures, around the realistic $T_{\text {str }} \sim 100$ K.

\section{B: Orbital polarization in the single-hole-pocket FeSe model}

In FeSe, the main effect of the SOI on the hole FSs is that the inner pocket sinks under the Fermi level. Similar bandstructure and the FS topology are realized by shifting the $d_{x y}$-orbital level at $\Gamma$-point by $-0.6 \mathrm{eV}$, as shown in Fig. S3. Based on this "single-hole-pocket FeSe model", we perform the numerical study of the orbital ordered state.
FeSe (single-hole-pocket model)
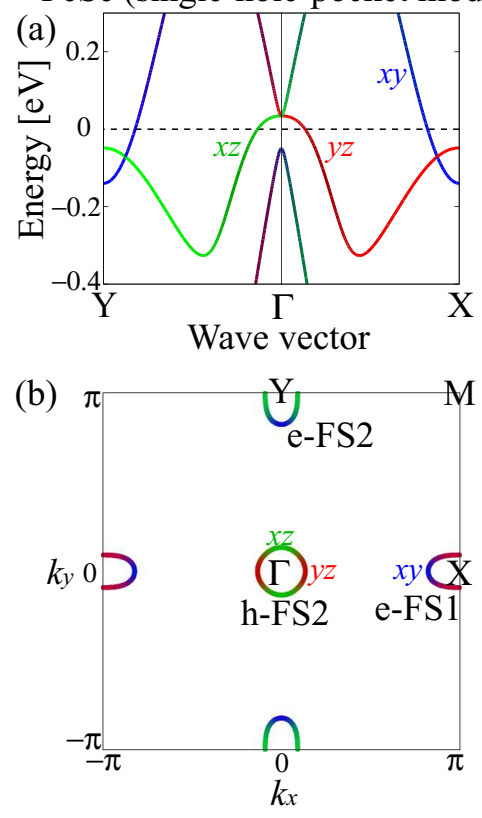

FIG. S3: (color online) (a) Bandstructure and (b) FSs in the single-hole-pocket FeSe model, by shifting the $d_{x y}$-orbital level at $\Gamma$-point by $-0.6 \mathrm{eV}$.

In the present model, the nematic orbital order is obtained for $\alpha_{S}>0.846(r>0.253)$. In Fig. S4, we show (a) the orbital polarization $\left(\Delta E_{x z}(\boldsymbol{k}), \Delta E_{y z}(\boldsymbol{k})\right)$ and (b) $C_{2}$ spin susceptibility $\chi^{s}(\pi, 0)>\chi^{s}(0, \pi)$ obtained for $\alpha_{S}=0.877$. Due to the orbital polarization, the size of the e-FS1 around X-point is reduced, and two Dirac-cone Fermi pockets appear. Another electron-pocket around Y-point, e-FS2, is enlarged. In addition, the single holepocket is elongated along the $k_{y}$-axis. Such FS deformation is essentially the same as that in the original FeSe model in the main text. In Figs. S4 (c)-(e), we show the $r$-dependences of the orbital orders, $\Delta n=n_{x z}-n_{y z}$, and $\alpha_{S}$, respectively.

In Fig. S5, we display the obtained bandstructures and orbital polarization for (a) $\alpha_{S}=0.865$ and (b) $\alpha_{S}=$ 0.877. In both cases, $\Delta E_{x z}(\mathrm{Y})-\Delta E_{y z}(\mathrm{X})$ is negative, whereas $\Delta E_{x z}(\Gamma)-\Delta E_{y z}(\Gamma)$ is positive. The obtained FSs are also shown in Figs. S5 (c) and (d), respectively. These results are almost the same as the results in Fig. 3 in the main text.

Thus, the sign-reversing orbital polarization in FeSe, which is the main result of the main text, is also realized in the single-hole-pocket FeSe model. This result indicates that the sign-reversing orbital order survives in the presence of the SOI. 

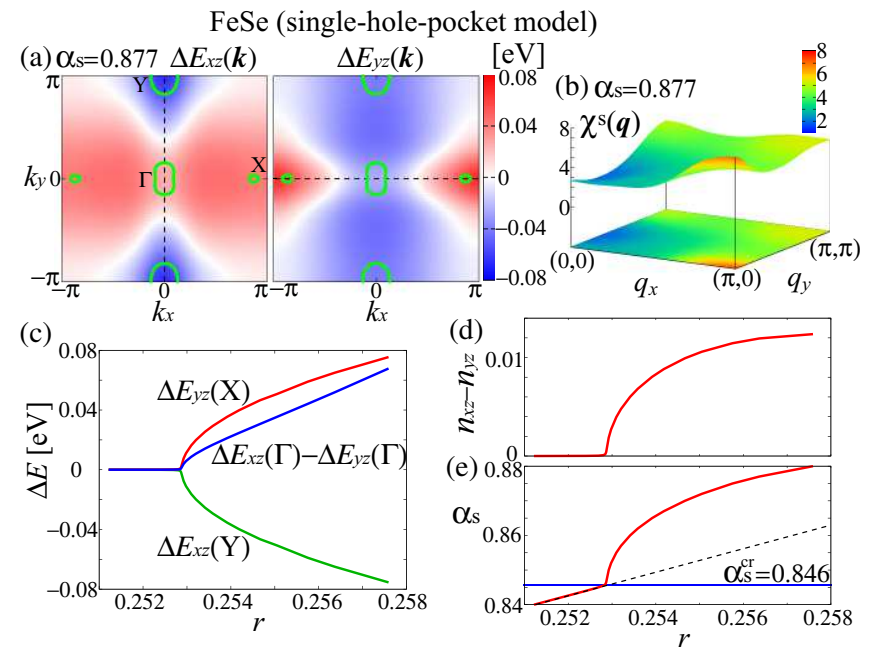

FIG. S4: (color online) (a) Obtained orbital polarization $\left(\Delta E_{x z}(\boldsymbol{k}), \Delta E_{y z}(\boldsymbol{k})\right)$ and (b) spin susceptibility in the singlehole-pocket FeSe model at $\alpha_{S}=0.877(r=0.256)$. The realized FSs are shown by the green lines. (c) The orbital polarization, (d) $\Delta n \equiv n_{x z}-n_{y z}$, and (e) $\alpha_{S}$ for $0.258>r>0.251$.
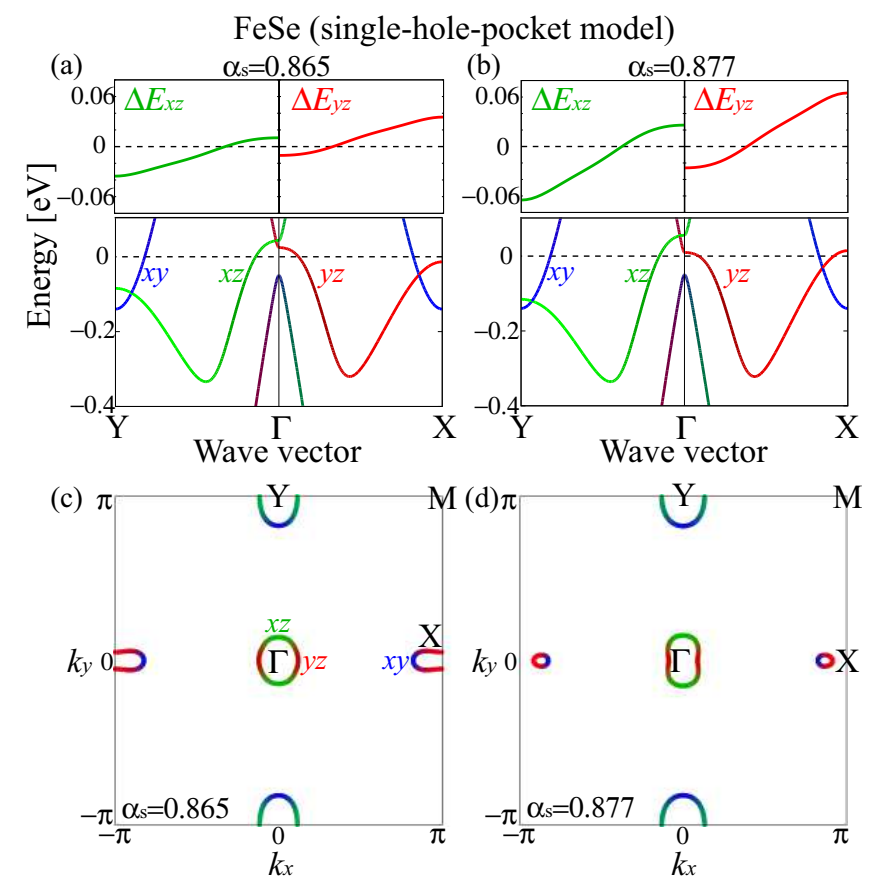

FIG. S5: (color online) Orbital polarizations and bandstructures in the single-hole-pocket FeSe model along $\mathrm{Y} \rightarrow \mathrm{\Gamma} \rightarrow \mathrm{X}$ for (a) $\alpha_{S}=0.865$ and (b) $\alpha_{S}=0.877$. The corresponding FSs are shown in (c) and (d), respectively.

\section{C: Comment on the self-consistent vertex correction (SC-VC) method}

In Ref. [27], the present authors studied various model Hamiltonians for Fe-based superconductors using the SCVC theory. In the FeSe model with $z_{4}^{-1}=3$, The critical spin Stoner factor for the orbital order is $\alpha_{S}^{\mathrm{cr}}=0.86$ at $T=50 \mathrm{meV}$. In the LaFeAsO model with $z_{4}^{-1}=1$ without the $d$-orbital level shifts, $\alpha_{S}^{\text {cr }}=0.975$. These critical spin Stoner factors are slightly larger than those obtained by the $C_{2}$ self-energy study in the main text. In FeSe, the orbital order is realized even when the spin fluctuations are weak in FeSe, due to the smallness of the ratio $\bar{J} / \bar{U}$ and the broadness of the $d_{x z / y z}$-orbital spin susceptibility, as explained in Ref. [27].

Here, we present an analytic explanation that the present self-consistent $C_{2}$ self-energy analysis is essentially equivalent to the SC-VC theory. In the main text, Fig. 4 (a) shows the diagrammatic expression of the selfenergy in Eq. (3), by expanding the right-hand-side with respect to $\Delta E_{l}=\Delta \Sigma_{l}$ : (i), (ii), and (iii), (iv) corresponds to the Hartree term, the Maki-Thompson (MT) term, and the AL-terms, respectively. In deriving Fig. 4 (a), we used the relation $\hat{G}=\hat{G}^{\Delta \mathrm{E}=0}+\hat{G}^{\Delta \mathrm{E}=0} \cdot \Delta \hat{E}$. $\hat{G}^{\Delta \mathrm{E}=0}+O\left(\Delta E^{2}\right)$. Based on Fig. 4 (a), the linearized self-consistent equation for $\Delta E_{l}(l=2,3)$ is obtained by introducing the eigenvalue $\lambda_{\text {orb }}$ as

$$
\begin{aligned}
& \lambda_{\text {orb }} \Delta E_{l}(\boldsymbol{k}) \approx T \sum_{k}\left[U\left\{G_{l}(k)\right\}^{2} \Delta E_{l}(\boldsymbol{k})\right. \\
& \left.\quad+\left(2 U^{\prime}-J\right)\left\{G_{5-l}(k)\right\}^{2} \Delta E_{5-l}(\boldsymbol{k})\right] \\
& +T \sum_{q} \frac{3}{2} V_{l}^{s}(q)\left\{G_{l}(k+q)\right\}^{2} \Delta E_{l}(\boldsymbol{k}+\boldsymbol{q}) \\
& +T \sum_{q}\left(G_{l}(\boldsymbol{k}+q)+G_{l}(\boldsymbol{k}-q)\right) \frac{3}{2}\left\{V_{l}^{s}(q)\right\}^{2} \Lambda_{l}(\Delta E ; q),
\end{aligned}
$$

where the first two lines give the intra-orbital and interorbital Hartree terms, respectively, and the third and fourth lines give the MT and AL terms, respectively. Here, we assumed the orbital-diagonal $\hat{G}$ and $\hat{V}^{s}$ for $\Delta \hat{E}=0$, and $\Lambda_{l}(\Delta E ; q)=-T \sum_{p}\left\{G_{l}(p)\right\}^{2} G_{l}(p+$ q) $\Delta E_{l}(\boldsymbol{p})$ is the three-point vertex. The eigenvalue $\lambda_{\text {orb }}$ exceeds unity when the orbital order is realized.

First, we explain that the orbital order mainly originates from the AL term: We put $\Delta E_{x z}(\boldsymbol{k})=$ $-\Delta E_{y z}(\boldsymbol{k})=\Delta E$ to simplify the discussion, and multiply both sides of Eq. (S3) by $-T \sum_{k}\left\{G_{l}(k)\right\}^{2}$ by dropping the MT term. Then, we obtain

$$
\lambda_{\text {orb }} \chi^{0}(\mathbf{0}) \approx\left(2 U^{\prime}-J-U\right)\left\{\chi^{0}(\mathbf{0})\right\}^{2}+X^{c}(\mathbf{0}),
$$

where $\chi^{0}(\mathbf{0})$ is the bare-bubble and $X^{c}(\mathbf{0})$ is the chargechannel AL-VC for $l=2$ or 3 . Thus, the orbital or$\operatorname{der}\left(\lambda_{\text {orb }}=1\right)$ is realized when $X^{c}(\mathbf{0})>\chi^{0}(\mathbf{0})-(U-$ $5 J)\left\{\chi^{0}(\mathbf{0})\right\}^{2} \sim 5 J / U^{2}$ for $U=U^{\prime}+2 J$, if we put $\chi^{0}(\mathbf{0}) \sim 1 / U$ for a qualitative estimation. This result is consistent with the condition for $\alpha_{C}=1$ in the $\mathrm{SC}-\mathrm{VC}$ theory $X^{c}(\mathbf{0})=(U-5 J)^{-1}-\chi^{0}(\mathbf{0}) \sim 5 J / U^{2}$ for $J / U \ll 1$ [27]. Thus, the present theory is a natural extension of the SC-VC theory for the orbital-ordered state.

We also explain that the MT term gives the signreversing orbital polarization in FeSe as explained in the 
main text. Considering the fact that $V_{3,3 ; 3,3}^{\Delta E=0}(\boldsymbol{q})$ has the peak at $(\pi, 0)$, the $\Delta E$-linear MT term in Eq. (S3) for $d_{y z}$-orbital at $\boldsymbol{k} \approx \mathbf{0}$ is approximately given as

$$
\begin{aligned}
& -\sum_{\boldsymbol{q}} V_{3,3 ; 3,3}^{\Delta E=0}(\boldsymbol{q}) \delta\left(E_{\boldsymbol{k}+\boldsymbol{q}}-\mu\right) \Delta E_{y z}(\boldsymbol{k}+\boldsymbol{q}) \\
& \quad \approx-\left\{\sum_{\boldsymbol{q}} V_{3,3 ; 3,3}^{\Delta E=0}(\boldsymbol{q}) \delta\left(E_{\boldsymbol{q}}-\mu\right)\right\} \Delta E_{y z}(\pi, 0)(\mathrm{S} 5)
\end{aligned}
$$

where $E_{k}$ is the $y z$-orbital band along $\Gamma$-X shown in Fig. 1 (a) in the main text, and we used the relation $T \sum_{n}\left(i \epsilon_{n}+\mu-E_{\boldsymbol{k}}^{\alpha}\right)^{-2}=-\delta\left(E_{\boldsymbol{k}}^{\alpha}-\mu\right)$ at $T \approx 0$. Therefore, the MT term tends to induce the sign reversal of
$\Delta E_{y z(x z)}(\boldsymbol{k})$ along the $k_{x(y)}$-axis, as depicted in the right inset of Fig. 4 (b) in the main text. Since $\Delta E_{y z}^{\mathrm{AL}}-\Delta E_{x z}^{\mathrm{AL}}$ is the smallest at $\Gamma$-point in FeSe, the sign-reversing orbital polarization is realized by the MT term.

In the present $C_{2}$ self-energy study, the higher-order MT- and AL-terms are included, and therefore the obtained $\alpha_{S}^{\text {cr }}$ is slightly smaller than that given by the SC$\mathrm{VC}$ theory. On the other hand, the feedback from the enhanced orbital susceptibility given by the $\mathrm{VC}$ is absent in the $C_{2}$ self-energy study. We will explain the relation between the $C_{2}$ self-energy theory and the SC-VC theory in more detail in future publications. 